\section{Choroidal thickness in regressed retinopathy of prematurity}

MF Anderson, B Ramasamy, DT Lythgoe and D Clark

\author{
Abstract \\ Purpose To compare choroidal thickness in \\ patients with regressed retinopathy of \\ prematurity (ROP) with healthy controls \\ using enhanced depth imaging optical \\ coherence tomography (EDI OCT) \\ Methods Twenty-four children and young \\ adults (41 eyes) with regressed $R O P \geq$ stage 3 \\ had undergone EDI OCT with Spectralis \\ FD-OCT as part of their clinical record. \\ Their refraction, best-corrected visual \\ acuity, and ophthalmoscopic findings were \\ recorded. Corresponding data was collected \\ prospectively from 33 healthy controls (58 \\ eyes) who had been born at term. Choroidal \\ thickness was measured independently by \\ two observers subfoveally and at $1500 \mu \mathrm{m}$ \\ nasal and temporal to the fovea using EDI \\ OCT. \\ Results Mean subfoveal choroidal \\ thickness, adjusted for refraction, was \\ 271.1 $\mu \mathrm{m}$ (95\% CI, 247.8-294.5) in the ex-ROP \\ group, which was significantly thinner than \\ $327.4 \mu \mathrm{m}$ (95\% CI, 293.8-360.9) in controls \\ $(P=0.008)$. Similarly, mean adjusted \\ temporal choroidal thickness was $257.2 \mu \mathrm{m}$ \\ (95\% CI, 240.2-274.2) in ex-ROP's vs $320.5 \mu \mathrm{m}$ \\ (95\% CI, 288.6-352.3) in controls $(P=0.001)$. \\ There was no statistically significant \\ difference in the nasal measurement. In the \\ ex-ROP group, there was no significant \\ correlation between subfoveal choroidal \\ thickness and gestational age $\left(r_{\mathrm{s}}=0.16\right.$, \\ $P=0.46)$ or birthweight $\left(r_{\mathrm{s}}=0.03, P=0.90\right)$. \\ In eyes without copathology in addition to \\ regressed ROP (29 eyes, 19 patients), there \\ was no significant correlation between \\ subfoveal choroidal thickness and visual \\ acuity. \\ Conclusions Our findings of thinner \\ subfoveal and temporal macular choroidal \\ thickness in regressed ROP support the case \\ for choroidal involvement in the \\ pathogenesis of this condition.
}

Eye (2014) 28, 1461-1468; doi:10.1038/eye.2014.207; published online 3 October 2014

Introduction

Retinopathy of prematurity (ROP) is a proliferative retinopathy affecting premature infants, which is characterized by abnormal maturation of the retinal vasculature.

Long-term visual outcomes are variable in patients with regressed ROP, even in the absence of macular pathology on ophthalmoscopy. Eyes in the Cryo-ROP study with regressed threshold disease had poorer visual acuity at 10 years than non-ROP preterm controls, despite normal-appearing posterior poles. ${ }^{1}$ The authors suggested that one explanation for the variability in vision might be non-retinal visual system abnormalities.

Prematurity without ROP has also been shown to affect vision. A study by Dowdeswell et $a l^{2}$ showed visual deficits in prematurely born children without ocular or cerebral pathology. Similarly, Larsson et $a l^{3}$ demonstrated poorer visual acuity in 10-year-old premature children, despite the exclusion of those who had ROP or neurologic disorders. It could not be established if the cause was retinal or posterior visual pathway related. O'Connor et al ${ }^{4}$ found poorer vision in low birthweight children than controls and found cases with reduced binocular acuity with no apparent ocular pathology.

Functional sequelae of ROP, despite normal macular appearance, have also been evidenced by electroretinography. Multifocal ERG showed abnormalities in the central maculae of eyes with spontaneously regressed ROP. ${ }^{5}$

Optical coherence tomography (OCT) is an imaging modality that has revealed new insights into the effect of ROP and/or prematurity on macular anatomy. Studies have shown shallowing of the foveal depression, thickening of the central macula, and a
Department of

Ophthalmology, University Hospital Aintree, Liverpool, UK

Correspondence:

MF Anderson, Department of Ophthalmology, Flinders Medical Centre, 1 Flinders Drive, Adelaide, SA 5042, Australia

Tel: +61 88204 4252;

Fax: +61 884042040 .

E-mail: matthewanderson2@ nhs.net

Received: 5 February 2014 Accepted in revised form: 31 July 2014

Published online:

3 October 2014

The findings of this study were presented in a poster at the Royal College of Ophthalmologists Annual Congress, 2013. 
continuity of inner retinal layers across the fovea in those born severely preterm, often despite normal macular appearance on ophthalmoscopy. ${ }^{6-14}$ However, these macular changes evident on OCT are still compatible with good visual acuity. $6,7,9-11$ This has prompted authors to suggest that other factors might be contributory to the variation in vision seen in these patients. ${ }^{10}$

Enhanced depth imaging (EDI) is an OCT technique developed for improved imaging of the choroid, whereby deeper ocular structures are placed closer to zero delay. EDI yields reproducible choroidal thickness (CT) measurements. ${ }^{15-20}$

Recent work on oxygen-induced retinopathy in an animal model has implicated choroidal involvement in the pathogenesis of ROP. ${ }^{21}$ The authors demonstrated sustained central choroidal involution in rats exposed to postnatal hyperoxia. Possible corroboration of this finding is offered by the work carried out by Park and $\mathrm{Oh}{ }^{22}$ who showed thinner choroid at the temporal macula in prematurely born children compared with controls using EDI OCT.

To investigate choroidal involvement in ROP and its sequelae, we used EDI OCT to measure CT in subjects with regressed ROP and compare this with controls.

\section{Materials and methods}

Children and young adults followed up in our department since infancy, after treatment for ROP, were invited to participate in this comparative case series. These patients were born with gestational age $<32$ weeks and/or birthweight $<1500 \mathrm{~g}$. Their retinopathy had been staged according to the International Classification of Retinopathy of Prematurity, and they had received treatment with laser (blue-green argon or $810 \mathrm{~nm}$ diode) and/or cryotherapy for ROP $\geq$ stage 3 with plus disease. ${ }^{23}$ Patients had been treated between February 1990 and July 1998.

The medical records of these patients were reviewed to obtain relevant demographic data and birth data. Information on staging and treatment of ROP was recorded, and any other ophthalmic pathology during their follow-up was noted. These patients had undergone OCT scanning, including EDI imaging, as part of their clinical record. Their spectacle refraction, best-corrected Snellen visual acuity, and the ophthalmoscopic findings at the time of the OCT imaging were recorded.

Eyes were excluded if there was pathology evident on ophthalmoscopy that might alter macular anatomy, such as dragging of the macula or vessels. Eyes with ocular copathology not affecting macular structure, such as strabismic amblyopia, were included only for analyses that did not involve visual acuity. In addition, eyes for which EDI images could not be obtained, or for which images were of insufficient quality to determine the choroidal boundaries (due to poor cooperation, inadequate fixation), were excluded.

A control group of children and young adults, who had been born at term ( $\geq 37$ weeks), was recruited prospectively from student volunteers and relatives of staff at our hospital. Participants underwent spectacle refraction and best-corrected Snellen acuity was recorded. Fundoscopy was performed, followed by OCT imaging in which EDI images were acquired. Control eyes had healthy maculae on ophthalmoscopy.

Amblyopic eyes were included for analyses that did not involve visual acuity. Eyes for which the choroidal boundaries could not be determined on EDI images were excluded from the control group.

Spectral domain OCT was performed using the Spectralis FD-OCT (Spectralis HRA-OCT; Heidelberg Engineering, Heidelberg, Germany). The technique of EDI OCT has been described previously. ${ }^{20}$ For the majority of cases, EDI images were generated by advancing the OCT device closer to the subject to obtain an inverted image, bringing deeper structures closer to zero delay. During the study, a software update, which automatically inverts the capturing screen and positions zero delay inferiorly instead of superiorly, allowed EDI images to be generated without performing the above manoeuvre. Images were acquired by experienced technicians in a dim room without pupil dilatation, using high-resolution mode. The eye tracking feature was used and multiple B scan averaging was used to increase signal-to-noise ratio and yield higher quality images. An internal fixation target was used, with the location of the scan on the retina monitored using the scanning laser ophthalmoscope image, which is available simultaneously. Scans were performed using the default axial length and refractive error settings.

CT was measured independently by two observers. Measurements were made manually using calipers provided by the proprietary software (Heidelberg Eye Explorer software version 5.3.3.0, Heidelberg Engineering), and were taken from the outer aspect of the hyperreflective band corresponding to the base of the retinal pigment epithelium (RPE), to the sclerochoroidal interface, as shown in Figure 1. Measurements were made perpendicular to the RPE, subfoveally and at two eccentric points, $1500 \mu \mathrm{m}$ nasal and temporal to the fovea. Magnification was set at $200 \%$. The fovea was located by the foveal depression, thickening of the outer nuclear layer, and elevation in the hyperreflective line commonly reported to represent the inner segment/outer segment (IS/OS) junction. The observers' measurements differed by $\geq 15 \%$ of their mean at seven locations, which were resolved by consensus. Measurements for both groups were made over the same time period. 

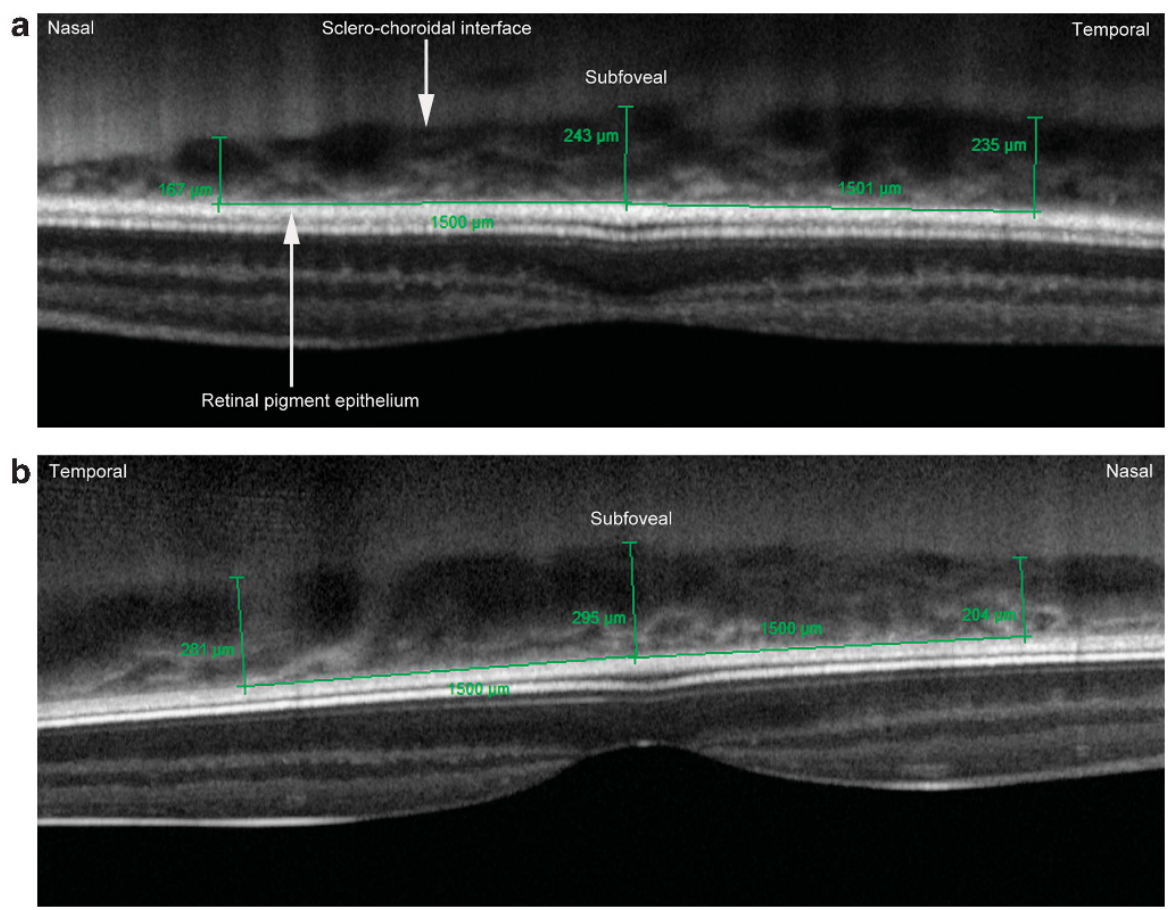

Figure 1 EDI OCT images showing measurement of CT subfoveally, and at $1500 \mu \mathrm{m}$ nasal and temporal to the fovea. (a) Left eye of a patient with regressed ROP. (b) Right eye of a control.

We compared CT measurements between cases and controls, and investigated the relationships between CT and gestational age, birthweight, refraction, and extent of ROP. When exploring the relationship between CT and visual acuity, we excluded those eyes with ocular copathology that could affect vision in the ex-ROP eyes (eg strabismic amblyopia, optic disc hypoplasia) and control eyes (eg strabismic amblyopia). Snellen visual acuity was converted to equivalent $\log$ MAR units for statistical analysis.

We certify that all applicable institutional and governmental regulations concerning the ethical use of human volunteers were followed during this research.

\section{Statistical methods}

The data in this study have a complex hierarchical structure with two observers making measurements, generally two eyes included per participant, and three measurement locations for each eye. As a result, standard independent sample statistical methods are generally not appropriate, as they are based on the assumption that observations are independent. Throughout the analysis, the data structure was appropriately accounted for using sophisticated statistical methods and/or the data structure was simplified. In simplifying we did the following: (1) used an average or consensus value instead of measurements from both observers; (2) in some parts of the analysis we averaged measurements across eyes. Pertaining to averaging across eyes, this is justified on the same grounds as Bland and Altman, that is, we were interested in between patient comparisons and used averaged values to represent each patient. ${ }^{24} \mathrm{We}$ conducted sensitivity analyses (not shown) where we analysed each location separately, effectively removing the problem of correlation between locations but retaining both eyes, and found negligible differences from the analyses used.

For demographic characteristics where there was a single observation per patient, standard independent sample $t$-tests and $\chi^{2}$-tests were used for continuous and categorical variables, respectively. Where there were two observations per patient (eg one per eye for visual acuity and refraction), linear regression with clustered robust standard errors was used to account for the inclusion of multiple observations per patient.

Spearman's rank correlation coefficients $\left(r_{\mathrm{s}}\right)$ were estimated using the method described by Bland and Altman on ranked data since multiple observations per patient were common. ${ }^{24}$ These correlation coefficients were compared using the method described by Cohen and Cohen. ${ }^{25}$

To compare subfoveal, nasal and temporal CTs both between and within groups, a covariance pattern linear mixed model was used. To simplify this analysis, CTs were averaged across eyes for those patients contributing 
more than one observation. The suitability of refraction as a covariate was assessed by including relevant twoway interaction terms.

Statistical analyses were undertaken using Stata version 12 (StataCorp. 2011. Stata Statistical Software: Release 12; StataCorp LP, College Station, TX, USA) and SAS/STAT 9.3 (SAS Institute Inc., Cary, NC, USA).

\section{Results}

In the regressed ROP group, 30 patients were recruited. Four patients were excluded because EDI OCT images had not been obtained because of logistical considerations. Two patients were excluded because of macular dragging complicating the ROP. In the remaining 24 patients, six eyes were excluded because of an inability to acquire satisfactory quality EDI images, and one eye was excluded because of an epiretinal membrane causing macular traction. Therefore, the cohort comprised 41 eyes, of which 20 eyes (12 patients) were from female patients. All eyes had been treated for stage 3 ROP in zone 2 with plus disease, except for: one eye without plus disease, which was not treated; one eye with stage $4 \mathrm{~A}$ ROP in zone 2; one eye with stage 3 and plus disease that was not treated. Twenty-eight eyes were treated with laser, 10 eyes with cryotherapy, and 1 eye with both modalities.

Thirty-four participants were recruited for the control group. One participant was excluded because the sclerochoroidal interface could not be identified on the EDI scans for either eye. In the remaining 33 participants, 3 eyes were excluded because it was not possible to discern the sclerochoroidal interface and 3 eyes were excluded because of technical scan errors (decentration, boundary errors). The cohort therefore comprised 60 eyes, of which 30 eyes (16 participants) were from female participants.

The characteristics of the study and control groups are summarized in Table 1.

Best-corrected visual acuity was significantly poorer in the ex-ROP group (mean logMar 0.06, SD 0.17) than in controls (mean logMar -0.07 , SD 0.11) $(P<0.0005)$. This included eyes with ocular copathology, such as amblyopia or nystagmus, which would affect visual acuity. When excluding eyes with such copathology, mean visual acuity was still poorer in cases (mean logMar -0.02 , SD 0.12) than in controls (mean logMar -0.09 , SD 0.06) $(P=0.02)$.

Pertaining to agreement of CT measurements between observers, the mean of the absolute difference between observers was $9.21 \mu \mathrm{m}$ (SD 13.27). The median EDI OCT scan quality was 36 (range 14-46, mean 34.10 \pm 8.09 ).

Figure 2 and Table 2 show that mean subfoveal and temporal CTs, adjusted for refraction, were significantly thinner in ex-ROP patients than in controls. The nasal measurement was thinner in ex-ROP patients, but this was not statistically significant. Refraction was found to be a valid covariate as two-way interactions between the factor variables group and choroid were not found to be statistically significant.

Figure 2 shows that mean subfoveal CT $>$ temporal $\mathrm{CT}>$ nasal CT in both groups. In the control group, the nasal CT was significantly thinner than both subfoveal CT $(P<0.0001)$ and temporal CT $(P<0.0001)$, whereas in

Table 1 Demographic data of the patient and control groups

\begin{tabular}{|c|c|c|c|}
\hline & $\begin{array}{c}\text { Cases } \\
\text { (41 eyes, } 24 \text { patients) }\end{array}$ & $\begin{array}{c}\text { Controls } \\
\text { (60 eyes, } 33 \text { patients) }\end{array}$ & P-value \\
\hline \multirow[t]{3}{*}{ Age (years) } & $15.2 \pm 2.84$ & $17.3 \pm 3.21$ & 0.015 \\
\hline & Median 16 & 17 & \\
\hline & Range 11-19 & $12-24$ & \\
\hline M:F & $12: 12$ & $16: 17$ & 0.91 \\
\hline \multirow[t]{2}{*}{ Gestational age (weeks) } & $26 \pm 1.75$ & $40 \pm 1.54$ & $<0.00005$ \\
\hline & Range $23-30$ & $37-42.14$ & \\
\hline \multirow[t]{2}{*}{ Birthweight (g) } & $854 \pm 191$ & $3598 \pm 496$ & $<0.00005$ \\
\hline & Range 512-1190 & $2296-4678$ & \\
\hline Spherical equivalent (D) & $-2.73 \pm 4.19$ & $-0.35 \pm 2.80$ & 0.02 \\
\hline \multirow[t]{2}{*}{ Visual acuity } & All eyes: $0.06 \pm 0.17$ & $-0.07 \pm 0.11$ & $<0.0005$ \\
\hline & Subgroup $^{\mathrm{a}}$ : $-0.02 \pm 0.12$ & $-0.09 \pm 0.06^{\mathrm{b}}$ & 0.02 \\
\hline \multicolumn{4}{|l|}{ Treatment (eyes) } \\
\hline Laser & 28 & & \\
\hline Cryotherapy & 10 & & \\
\hline Both & 1 & & \\
\hline Untreated & 2 & & \\
\hline
\end{tabular}

aSubgroup refers to regressed ROP eyes without ocular copathology that could affect visual acuity ( $n=29$ eyes).

${ }^{\mathrm{b}}$ Refers to control eyes without amblyopia $(n=58)$. 
the ex-ROP group the nasal CT was thinner than subfoveal CT $(P=0.0002)$ but not temporal CT $(P=0.2)$.

For the subset of ex-ROP eyes without ocular copathology expected to affect vision (29 eyes, 19 patients), there was no significant correlation between subfoveal CT and best-corrected visual acuity $\left(r_{\mathrm{s}}=-0.09, P=0.73\right)$. There was similarly no correlation for controls without amblyopia $\left(r_{\mathrm{S}}=-0.18, P=0.33\right)$

(58 eyes, 33 participants).

In the ex-ROP cohort, a positive correlation was found between spherical equivalent and subfoveal CT $\left(r_{\mathrm{S}}=0.57\right.$, $P=0.003$ ), such that more myopic eyes tended to have thinner choroids. This relationship was also demonstrated for nasal $\left(r_{\mathrm{s}}=0.44, P=0.03\right)$ and temporal ( $\left.r_{\mathrm{S}}=0.65, P=0.0006\right)$ locations. However, in the control group, no such correlation was found at any location ( $\left.r_{\mathrm{s}}=0.13, P=0.48\right)$ for the subfoveal location).

In the ex-ROP group, there was no significant correlation between subfoveal CT and gestational age $\left(r_{\mathrm{s}}=0.16, P=0.46\right)$. There was also no significant correlation between subfoveal CT and birthweight in the ex-ROP $\left(r_{\mathrm{s}}=0.03, P=0.90\right)$ or the control group $\left(r_{\mathrm{s}}=0.34\right.$, $P=0.053)$.

There was a negative correlation between the extent of ROP, measured in clock hours, and unadjusted subfoveal CT $\left(r_{\mathrm{s}}=-0.52, P=0.009\right)$. A similar relationship was apparent at the nasal $\left(r_{\mathrm{s}}=-0.44, P=0.03\right)$ and temporal

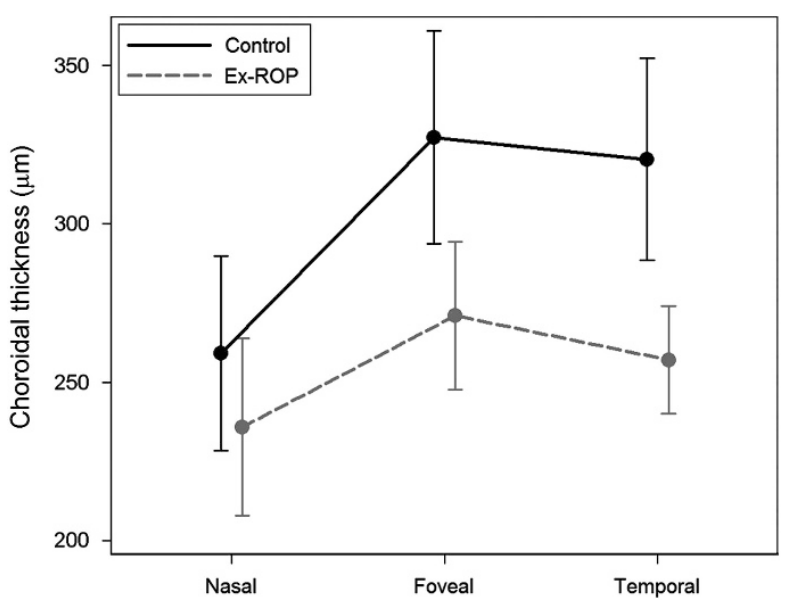

Figure 2 Choroidal thickness, adjusted for refractive error, in the regressed ROP and control groups. locations, although at the latter the correlation was not statistically significant $\left(r_{\mathrm{s}}=-0.39 . P=0.06\right)$. This analysis excluded the two untreated eyes, and the eye with stage $4 \mathrm{~A}$, so that $n=38$ eyes ( 23 patients).

\section{Discussion}

Although the ophthalmoscopic vascular changes in ROP are most evident in the retinal periphery, the visual deficits associated with regressed ROP or premature birth point to central retinal dysfunction. ${ }^{1-4,12-14}$ Electroretinography also suggests that central retinal dysfunction is greater than peripheral dysfunction. ${ }^{5}$ OCT has revealed changes not evident on ophthalmoscopy, but these can be compatible with good visual acuity. ${ }^{6,7,9-11}$ Authors have questioned the possibility of a non-retinal aetiology for visual deficits, so examination of the choroid is warranted. ${ }^{1}$

The choroid serves functions including metabolic support for the RPE and retina, acting as a heat sink, and absorbing excess light. ${ }^{26,27}$ Abnormalities of the choroid or CT have been demonstrated in a number of disease states, including exudative age-related macular degeneration, idiopathic polypoidal choroidal vasculopathy, central serous retinopathy, Vogt-Koyanagi-Harada disease, and high myopia. ${ }^{28-32}$ Previous work using OCT has established that CT is positively correlated with refraction, negatively correlated with axial length, and that CT declines with age. ${ }^{16-19,33-35}$

Evidence of prematurity affecting central, and not only peripheral, vasculature was reported by Mintz-Hittner et $a l^{36}$ who showed a reduced foveal avascular zone in prematurely born children. This finding was compatible with normal vision.

Choroidal involvement has been implicated in the pathogenesis of ROP using a rat model of oxygeninduced retinopathy. ${ }^{21}$ Shao et $a l^{21}$ demonstrated sustained choroidal involution, predominantly in the central fundus, in rats exposed to postnatal hyperoxia. The authors found that elevated levels of $15 \mathrm{~d}-\mathrm{PGJ}_{2}$ in the central fundus induced apoptosis of ocular endothelial cells through the induction of oxidant stress. They suggested that this choroidal degeneration could explain the central functional deficits associated with ROP.

Table 2 Mean choroidal thickness values, adjusted for refractive error, in the regressed ROP and control groups

\begin{tabular}{lcccc}
\hline & Cases (95\% CI) & Controls $(95 \%$ CI $)$ & $\begin{array}{c}\text { Differences } \\
\text { controls - cases (95\% CI) }\end{array}$ & P-value \\
\hline Subfoveal CT & $271.1(247.8 ; 294.5)$ & $327.4(293.8 ; 360.9)$ & $56.3(15.2 ; 97.3)$ & 0.008 \\
Temporal CT & $257.2(240.2 ; 274.2)$ & $320.5(288.6 ; 352.3)$ & $63.3(27.1 ; 99.5)$ & 0.001 \\
Nasal CT & $236.0(208.0 ; 263.9)$ & $259.3(228.6 ; 290.0)$ & $23.3(-18.6 ; 65.3)$ & 0.27 \\
\hline
\end{tabular}


Recently, Park and $\mathrm{Oh}^{22}$ found $\mathrm{CT}$, adjusted for age and axial length, to be significantly thinner at a location $3 \mathrm{~mm}$ temporal to the fovea in prematurely born children compared with controls. A marginal negative correlation between stage of ROP and CT at this site was found. No correlation between visual acuity and CT was demonstrated, and there was no significant difference in CT between the groups at other sites.

Our results show that subfoveal and temporal CT in patients with regressed ROP is significantly thinner than in term controls. We found subfoveal CT, adjusted for refraction, to be on average $56.3 \mu \mathrm{m}(95 \% \mathrm{CI}$, 15.2-97.3) thinner in the ex-ROP cohort. This finding supports the case for choroidal involvement in the pathogenesis of ROP as proposed by Shao et al, ${ }^{21}$ and could be in keeping with choroidal involution as reported by them.

It is possible that the choroidal thinning we observed is a consequence of the treatment for ROP rather than the condition itself, or because of an unmeasured factor. We could not recruit an untreated cohort with ROP of this severity, given that cases meet the criteria for treatment. It is noted, however, that the choroidal thinning observed by Park and $\mathrm{Oh}^{22}$ did not correlate with laser treatment in their study.

The mean unadjusted subfoveal CT measurement for the control group in our study $(338.0 \mu \mathrm{m})$ was in agreement with other reported studies. 15,18-20,22,33,35

While mean subfoveal and temporal CT was statistically significantly thinner in ex-ROP patients than controls, the clinical significance of this finding needs to be interpreted in the context of a wide reported range of CT in normals. The subfoveal $\mathrm{CT}$ in our control group, for example, ranged from 145 to $549 \mu \mathrm{m}$. Indeed, the mean CT as measured by OCT in normals in other studies has ranged from 203.6 to $448 \mu \mathrm{m} .{ }^{17,37}$ Chen et al ${ }^{18}$ showed the absolute interocular subfoveal CT difference can be as high as $135 \mu \mathrm{m}$ within individuals.

We did not demonstrate a correlation between $\mathrm{CT}$ and gestational age or birthweight. However, our finding of negative correlations between subfoveal and nasal CT and clock hours of ROP suggests that choroidal thinning is more marked in more severe ROP, in keeping with the finding of Park and Oh. ${ }^{22}$ Almost all eyes in our series received treatment for stage $3 \mathrm{ROP}$ in zone 2 associated with plus disease. If ROP were to be treated earlier, as currently may occur with treatment of type 1 prethreshold ROP, as defined in the Early Treatment for Retinopathy of Prematurity (ETROP) study, this might feasibly limit the reduction in CT observed. ${ }^{38}$ Treating earlier disease might attenuate any effect of $\mathrm{ROP}$ on $\mathrm{CT}$, and therefore on any sequelae.
Our finding of subfoveal CT $>$ temporal $\mathrm{CT}>$ nasal CT was in keeping with other studies. ${ }^{16,17,34}$ Interestingly, the nasal CT was significantly thinner than temporal CT in the control group, but not in cases, suggesting that the typical nasal thinning of the choroid is less marked in regressed ROP.

In the ex-ROP group, the positive correlation that we demonstrated between spherical equivalent and $\mathrm{CT}$ is in keeping with reports in the literature. ${ }^{19,33}$ We did not demonstrate the same relationship in the controls, which may be because of our sample size and/or the narrower range of refractive error in this group compared with the cases.

Fujiwara $e t a l^{32}$ demonstrated a negative correlation between $\mathrm{CT}$ and visual acuity in high myopia, and suggested a role for choroidal atrophy in the pathogenesis of visual dysfunction in this condition. Similarly, in a study investigating the cause of poor visual acuity in high myopia, Nishida et al ${ }^{39}$ found subfoveal CT to be negatively correlated with visual acuity. It was the only significant predictor of visual acuity in this group, and they suggested that a very thin choroid might deliver decreased metabolic support to the outer retina, which could affect photoreceptor function or cause photoreceptor loss.

We noted poorer visual acuity in the ex-ROP subgroup of eyes without associated ocular copathology than in controls. However, we did not demonstrate any correlation between visual acuity and subfoveal CT in either this ex-ROP subgroup or controls. Further work is needed to elucidate whether the thinner choroid in regressed ROP is a benign consequence of ROP or prematurity, or if it is contributory to the associated visual function deficits.

The limitations of our study include the retrospective method of data collection for the ex-ROP cohort. CT measurements were made manually and therefore may be susceptible to error, although good interobserver agreement was observed. Measurements at discrete sampling points may be influenced by focal thickening or thinning of the choroid, or irregularity of the chorioscleral border, thus it is possible that these points may not always be representative of the CT. ${ }^{30}$ We recorded refractive error, but it would be useful to investigate the effect of axial length in this setting in future work, particularly as high myopia in regressed ROP is associated with altered anterior segment development, a more globular lens, and increased corneal power, and not solely axial length. ${ }^{40,41}$ Furthermore, it would be beneficial for future studies to include a premature cohort without ROP to determine whether choroidal thinning is associated with ROP only or whether prematurity per se has an effect on CT. 


\section{Summary}

\section{What was known before}

- Long-term visual outcomes are variable in regressed ROP even in the absence of macular pathology on ophthalmoscopy.

- OCT has demonstrated central macular abnormalities in those born severely preterm, although these are compatible with good visual acuity. EDI OCT allows reproducible measurements of the choroid.

- Work on oxygen-induced retinopathy in an animal model has implicated choroidal involvement in the pathogenesis of ROP. Thinner choroid at the temporal macula in prematurely born children has been demonstrated with EDI OCT.

\section{What this study adds}

- This study has shown subfoveal CT, adjusted for refraction, to be significantly thinner in patients with regressed severe ROP than in controls.

- The extent of choroidal thinning appears to be related to the extent of ROP.

- These findings support the case for choroidal involvement in the pathogenesis of ROP, and possibly in the associated visual deficits.

\section{Conflict of interest}

The authors declare no conflict of interest.

\section{Acknowledgements}

We thank Mr Richard Hancock (Department of Ophthalmic and Clinical Photography, Aintree University Hospital), who facilitated acquisition of OCT images.

\section{References}

1 Dobson V, Quinn GE, Summers CG, Hardy RJ, Tung B. Cryotherapy for Retinopathy of Prematurity Cooperative Group. Visual Acuity at 10 Years in Cryotherapy for Retinopathy of Prematurity (CRYO-ROP) Study Eyes. Arch Ophthalmol 2006; 124: 199-222.

2 Dowdeswell HJ, Slater AM, Broomhall J, Tripp J. Visual deficits in children born at less than 32 weeks' gestation with and without major ocular pathology and cerebral damage. Br J Ophtalmol 1995; 79: 447-452.

3 Larsson EK, Rydberg AC, Holmstrom GE. A populationbased study on the visual outcome in 10-year old preterm and full-term children. Arch Ophthalmol 2005; 123: 825-832.

4 O'Connor AR, Stephenson TJ, Johnson A, Tobin MJ, Ratib S, Moseley $\mathrm{M}$ et al. Visual function in low birthweight children. Br J Ophthalmol 2004; 88: 1149-1153.

5 Fulton AB, Hansen RM, Moskowitz A, Barnaby AM. Multifocal ERG in subjects with a history of retinopathy of prematurity. Doc Ophthalmol 2005; 111: 7-13.

6 Ecsedy M, Szamosi A, Karko C, Zubovics L, Varsanyi B, Nemeth $\mathrm{J}$ et al. A comparison of macular structure imaged by optical coherence tomography in preterm and full-term children. Invest Ophthalmol Vis Sci 2007; 48: 5207-5211.
7 Hammer DX, Iftimia NV, Ferguson RD, Bigelow CE, Ustun TE, Barnaby AM et al. Foveal fine structure in retinopathy of prematurity: an adaptive optics Fourier Domain Optical Coherence Tomography Study. Invest Ophthalmol Vis Sci 2008; 49: 2061-2070.

8 Baker PS, Tasman W. Optical coherence tomography imaging of the fovea in retinopathy of prematurity. Ophthalmic Surg Lasers Imag 2010; 41: 201-206.

9 Wang J, Spencer R, Leffler JN, Birch EE. Critical period for foveal fine structure in children with regressed retinopathy of prematurity. Retina 2012; 32: 330-339.

10 Recchia FM, Recchia CC. Foveal dysplasia evident by optical coherence tomography in patients with a history of retinopathy of prematurity. Retina 2007; 27: 1221-1226.

11 Akerblom H, Larsson E, Eriksson U, Holmstrom G. Central macular thickness is correlated with gestational age at birth in prematurely born children. Br J Ophthalmol 2011; 95: 799-803.

12 Tariq YM, Burlutsky G, Mitchell P. Macular parameters and prematurity: a spectral domain coherence tomography study. J AAPOS 2012; 16: 382-385.

13 Yanni SE, Wang J, Chan M, Carroll J, Farsiu S, Leffler JN et al. Foveal avascular zone and foveal pit formation after preterm birth. Br J Ophthalmol 2012; 96: 961-966.

14 Wu W, Lin R, Shih C, Wang N, Chen Y, Chao A et al. Visual acuity, optical components, and macular abnormalities in patients with a history of retinopathy of prematurity. Ophthalmology 2012; 119: 1907-1916.

15 Rahman W, Chen FK, Yeoh J, Patel P, Tufail A, Da Cruz L. Repeatability of manual subfoveal choroidal thickness measurements in healthy subjects using the technique of enhanced depth imaging optical coherence tomography. Invest Ophthalmol Vis Sci 2011; 52: 2267-2271.

16 Manjunath V, Taha M, Fujimoto JG, Duker JS. Choroidal thickness in normal eyes measured using Cirrus HD optical coherence tomography. Am J Ophthalmol 2010; 150: 325-329.

17 Hirata M, Tsujikawa A, Matsumoto A, Hangai M, Ooto S, Yamashiro $\mathrm{K}$ et al. Macular choroidal thickness and volume in normal subjects measured by swept-source optical coherence tomography. Invest Ophthalmol Vis Sci 2011; 52: 4971-4978.

18 Chen FK, Yeoh J, Rahman W, Patel PJ, Tufail A, Da Cruz L. Topographic variation and interocular symmetry of macular choroidal thickness using enhanced depth imaging optical coherence tomography. Invest Ophthalmol Vis Sci 2012; 53: 975-985.

19 Shin JW, Shin YU, Lee BR. Choroidal thickness and volume mapping by a six radial scan protocol on spectral-domain optical coherence tomography. Ophthalmology 2012; 119: 1017-1023.

20 Spaide RF, Koizumi H, Pozonni MC. Enhanced depth imaging spectral domain optical coherence tomography. Am J Ophthalmol 2008; 146: 496-500.

21 Shao Z, Dorfman AL, Seshadri S, Djavari M, Kermorvant-Duchemein E, Sennlaub F et al. Choroidal involution is a key component of oxygen-induced retinopathy. Invest Ophthalmol Vis Sci 2011; 52: 6238-6248.

22 Park K, Oh SY. Analysis of spectral-domain optical coherence tomography in preterm children: retinal layer thickness and choroidal thickness profiles. Invest Ophthalmol Vis Sci 2012; 53: 7201-7207.

23 The Committee for the Classification of Retinopathy of Prematurity. An International Classification of Retinopathy of Prematurity. Arch Ophthalmol 1984; 102: 1130-1134. 
24 Bland JM, Altman DG. Calculating correlation coefficients with repeated observations: Part 2-correlation between subjects. BMJ 1995; 310: 633.

25 Cohen J, Cohen P. Applied Multiple Regression/Correlation, Analysis for the Behavioral Sciences. 2nd edn. Lawrence Erlbaum Associates: Hillsdale, NJ, USA, 1983.

26 Linsenmeier RA, Padnick-Silver L. Metabolic dependence of photoreceptors on the choroid in the normal and detached retina. Invest Ophthalmol Vis Sci 2000; 41: 3117-3123.

27 Parver LM, Auker C, Carpenter DO. Choroidal blood flow as a heat dissipating mechanism in the macula. Am J Ophthalmol 1980; 89: 641-646.

28 Chung SE, Kang SW, Lee JH, Kim YT. Choroidal thickness in polypoidal choroidal vasculopathy and exudative age-related macular degeneration. Ophthalmology 2011; 118: 840-845.

29 Imamura Y, Fujiwara T, Margolis R, Spaide RF. Enhanced depth imaging optical coherence tomography of the choroid in central serous chorioretinopathy. Retina 2009; 29: 1469-1473.

30 Jirarattanasopa P, Ooto S, Tsujikawa A, Yamashiro K, Hangai M, Hirata $M$ et al. Assessment of macular choroidal thickness by optical coherence tomography and angiographic changes in central serous chorioretinopathy. Ophthalmology 2012; 119: 1666-1678.

31 Nakai K, Gomi F, Ikuno Y, Yasuno Y, Nouchi T, Ohguro N et al. Choroidal observations in Vogt-Koyanagi-Harada disease using high-penetration optical coherence tomography. Graefes Arch Clin Exp Ophthalmol 2012; 250: 1089-1095.

32 Fujiwara T, Imamura Y, Margolis R, Slakter JS, Spaide RF. Enhanced depth imaging optical coherence tomography of the choroid in highly myopic eyes. Am J Ophthalmol 2009; 148: $445-450$.
33 Goldenberg D, Moisseiev E, Goldstein M, Loewenstein A, Barak A. Enhanced depth imaging optical coherence tomography: choroidal thickness and correlations with age, refractive error, and axial length. Ophthalmic Surg Lasers Imaging 2012; 43: 296-301.

34 Margolis R, Spaide RF. A pilot study of enhanced depth imaging optical coherence tomography of the choroid in normal eyes. Am J Ophthalmol 2009; 147: 811-815.

35 McCourt EA, Cadena BC, Barnett CJ, Ciardella AP, Mandava N, Kahook MY. Measurement of subfoveal choroidal thickness using spectral domain optical coherence tomography. Ophthalmic Surg Lasers Imag 2010; 41: S28-S33.

36 Mintz-Hittner HA, Knight-Nanan DM, Satriano DR, Kretzer FL. A small foveal avascular zone may be an historic mark of prematurity. Ophthalmology 1999; 106: 1409-1413.

37 Benavente-Perez A, Hosking SL, Logan NS, Bansal D. Reproducibility-repeatability choroidal thickness calculation using optical coherence tomography. Optom Vis Sci 2010; 87: 867-872.

38 Good WV. Early Treatment for Retinopathy of Prematurity Cooperative Group. Final results of the Early Treatment for Retinopathy of Prematurity (ETROP) Randomized Trial. Trans Am Ophthalmol Soc 2004; 102: 233-250.

39 Nishida Y, Fujiwara T, Imamura Y, Lima LH, Kurosaka D, Spaide RF. Choroidal thickness and visual acuity in highly myopic eyes. Retina 2012; 32: 1229-1236.

40 Garcia-Valenzuela E, Kaufman L. High myopia associated with retinopathy of prematurity is primarily lenticular. J AAPOS 2005; 9: 121-128.

41 Gallo JE, Fagerholm P. Low-grade myopia in children with regressed retinopathy of prematurity. Acta Ophthalmol 1993; 71: 519-523. 